\title{
Haptic anchoring and human postural control
}

\author{
Eliane Mauerberg-deCastro ${ }^{1,2}$, Renato Moraes $^{2}$, Carolina Paioli Tavares ${ }^{1,3}$, Gabriella Andreeta \\ Figueiredo $^{2}$, Stephanie C.M. Pacheco ${ }^{1}$, and Thais D.A. Costa ${ }^{1}$ \\ 1- Universidade Estadual Paulista, Rio Claro, SP, Brazil \\ 2- Universidade de São Paulo, Ribeirão Preto, SP, Brazil \\ 3- Universidade Estadual de Ponta Grossa, Ponta Grossa, PR, Brazil
}

\begin{abstract}
Several studies have emphasized the contribution of haptic input that results from the use of rigid and non-rigid tools to the postural control system. Experimental protocols such as the light touch and the anchor system are based on individuals' haptic exploration of the environment through direct tactile-kinesthetic contact, or indirectly through rigid or flexible tools that are attached to the body. In this article, we introduce the main findings of humans' haptic use of non-rigid tools during postural control tasks. We illustrate the effects of an anchor system paradigm on the maintenance of stability via haptic information. Haptic anchoring includes the handling of flexible cables that are attached to loads that are in contact with a surface. We include results of studies about haptic information gathered during the holding of a walking dog's leash. Studies that used the anchor system demonstrated its effectiveness in reducing body sway in several groups, including young adults, children, older individuals, and intellectually disabled individuals. We discuss several experimental designs and intervention protocols in order to illustrate how haptic anchoring could prompt functional plasticity. Keywords: haptic perception, postural control, non-rigid tools, anchor system.
\end{abstract}

Received 06 January 2014; received in revised form 03 August 2014; accepted 12 August 2014. Available online 25 November 2014.

\section{Introduction}

Popularly defined as an individual's use of the sense of touch to recognize objects (Klatzky, Lederman, \& Metzger, 1985; Lederman \& Klatzky, 2009; Loomis \& Lederman, 1986), haptic perception is an action-perception system that detects information by dynamically combining input patterns from mechanoreceptors located close to the skin's surface and mechanoreceptors that are embedded in muscles, tendons, and joints (kinesthetic inputs). The muscle senses and muscle-generated force patterns are automatically integrated into a complex actionperception process that, depending on the nature of the task, may encompass tactile information via a voluntary control mechanism. Vision and audition, for example, are complemented by haptic inputs (Frissen, Ziat, Campion, Hayward, \& Guastavino, 2012). Additionally, haptic perception has evolved to include the detection of time-

Eliane Mauerberg-deCastro, Carolina Paioli Tavares, Stephanie C.M. Pacheco, and Thais D.A. Costa, Universidade Estadual Paulista. Eliane Mauerberg-deCastro, Renato Moraes, and Gabriella Andreeta Figueiredo, Universidade de São Paulo. Carolina Paioli Tavares, Universidade Estadual de Ponta Grossa. Correspondence regarding this article should be directed to: Eliane Mauerberg-deCastro, Laboratory of Action and Perception, Department of Physical Education, Universidade Estadual Paulista, Av. 24-A, 1515, Bela Vista, Rio Claro, SP, 13506-900, Brazil. E-mail: mauerber@rc.unesp.br invariant parameters that emerge from the spatiotemporal structure of ambient arrays (Cabe, 2011; Cabe \& Hofman, 2012; Gibson, 1966). While the individual elements of an experience with motion change (e.g., distance to targets, flow of visual field, etc.), the lawful properties of the mass distribution of muscles and objects at hand (e.g., inertia, moments of inertia, and vibratory resonance) remain perceptually constant or invariant (Carello, Silva, Kinsella-Shaw, \& Turvey, 2008; Carello \& Turvey, 2004; Michaels \& Carello, 1981).

This detection of invariants is possible as long as the environment affords ${ }^{1}$ possibilities for an action or behavior. The haptic system, via exploratory behavior, picks up information about changes in the relative position or the velocity of a handled object or one connected to moving body parts (patterns of energy) and transforms this "touched" world into a meaningful, functional relationship. For example, during the handling of tools that are used to recover relevant aspects of a medium and surfaces (e.g., surgical instruments used during surgery to capture details about tissue), a system of force patterns (i.e., torque, thrust, and drag)

\footnotetext{
${ }^{1}$ Affordance is a key concept in the ecological direct perception approach postulated by Gibson (1979). Affordance is the possibility for an organism, while constrained by a task goal, to perform an action or behavior as determined by an event, place, or object.
} 
provides the individual with information about the spatial configuration of a task context (surgery). In such instances, haptic perception is constrained by a multioriented and multi-scaled context that affords a solution to a (surgical) problem.

A multi-oriented and multi-scaled context can be illustrated in a system of linkages between relatively rigid structures and relatively non-rigid tensile structures that collectively ensure information flow across biological levels, from the cellular scale to the entire system, and beyond the body. Recently, in order to identify how the haptic system's perception operates by transferring energy patterns (i.e., information) through a biological system at multi-scale levels, such as between the constituent elements of muscular, connective, and skeletal tissue nets, Turvey and Fonseca (2014) developed a multifractal tensegrity hypothesis. The multifractal tensegrity hypothesis relies on the idea that, in the physical medium, propagation and distribution of mechanical disturbances (i.e., shape and stability) collectively flow across different biological scales (i.e., from cell deformation to the entire body). According to Turvey and Fonseca, with the continuous tension and discontinuous compression of cells, tissue nets (i.e., microscopic) become dynamic patterns that ultimately yield information at a behavioral scale (i.e., macroscopic).

According to Carello and Turvey (2004), when an individual handles an object, the imprinted pattern of forces on muscles, tendons, and ligaments results in accurate, metrically appropriate impressions (higherorder relations) of the spatial layout that is being explored. Therefore, haptic perception encompasses a complex interplay between lower- and higher-order systems and subsystems that collectively integrate information about events or properties that act on a perceiver. Indeed, researchers (Solomon \& Turvey, 1988; Turvey, 1996) have devoted extensive efforts to understanding haptic functioning, mostly in experiments that involve an individual's manipulation of objects that are used to estimate the dimensions of adjacent, outof-sight targets (e.g., probing the length of an object or distance to a target with some type of an extension rod). To capture or expand access to information about surfaces or media that are not in direct contact with an individual's innervated tissue, the use of objects or noninnervated structures depends on the affordances of a particular organism's ecological niche (Gibson, 1966).

Many examples in the animal world illustrate how non-innervated appendages are crucial to the haptic detection of energy patterns of a medium, object, or other animal (Burton, 1993). For example, some animals use antennae to pick up the air vibrations of approaching prey or predators. Humans have incorporated nonbiological appendages as part of their bodily functions. For example, missing limbs or dysfunctional body parts have been replaced by prostheses, orthoses, and implants for the purpose of facilitating mobility. In many cases related to human daily living activities, however, tools are incorporated into the activity, and this in itself characterizes a task (e.g., brushing hair, cutting paper with scissors, writing, and using silverware for eating).

Haptic perception also allows the reorganization of functions and information across perceptual modalities. When vision is unavailable during tasks that require an individual to determine magnitudes of a target or object layout, other perceptual modalities can provide relatively accurate details about the metrics of such objects (Turvey, 1996). Indeed, one can estimate the size of a fish caught in deep, cloudy water by maneuvering the fishing pole and reacting to the pulling/dragging patterns of forces that result from the fish struggling at the end of the line. A fisherman, besides anticipating the size of the fish, can also estimate the depth at which the fish is likely located throughout the chase, prior to the final catch. These skills can be quite accurate (and motivating) in an experienced fisherman. Such dynamic acquisition of information helps determine the behavioral strategies that regulate particular actions in a given context.

Additionally, haptic perception supplies the body's orientation system with information about its relative position in space (i.e., kinesthesis and skin receptors, or pick up of movement, according to Gibson, 1966). Because the body is constantly in motion due to internal metabolic activity, in order to sustain certain high-order activities (e.g., writing on a board while standing), regulatory mechanisms of posture reduce the levels of routine instability. For example, as an individual reads a message from a distant sign (i.e., supra-postural task), anticipatory control mechanisms quiet head oscillation in order for this view to be stabilized in the retina (Riccio \& Stoffregen, 1988; Riley, Stoffregen, Grocki, \& Turvey, 1999; Stoffregen, Smart, Bardy, \& Pagulayan, 1999). Such a mechanism is determined by visual kinesthetics and allows "perspective transformations of the field of view" (Gibson, 1966, p. 111).

Moreover, the relationship between the control of posture and haptics is illustrated when an individual lightly touches a surface to maintain balance. The canonical "light touch" experiment (Holden, Ventura, \& Lackner, 1994) showed that the somatosensory region of the subtle contact (typically the fingers) provides, in a very brief period of time, a reference frame for orientation of the body and its parts, which results in reduced postural sway. Such findings are quite robust and over the past 20 years have been consistently replicated in the literature (Bolton, McIlroy, \& Staines, 2011; Holden et al., 1994; Jeka, 1997; Jeka \& Lackner, 1994; Jeka, Schoner, Dijkstra, Ribeiro, \& Lackner, 1997; Lackner, Rabin, \& Dizio, 2001; Rabin, Dizio, \& Lackner, 2006; Riley et al., 1999; Vuillerme, Isableu, \& Nougier, 2006).

The expansion of haptic information as a means of helping the postural control system to achieve stability after perturbation has been observed in a variety of situations such as early in development (Barela, Jeka, \& Clark, 1999), during the suppression 
of vision (Jeka \& Lackner, 1994), impaired input due to vestibular loss (Horak, 2009), or other balanceimpaired conditions such as in older individuals or in neurological conditions that affect balance (Baccine et al., 2007; Baldan, Alouche, Araujo, \& Freitas, 2014; Rabin, Chen, Muratori, DiFrancisco-Donoghue, \& Werner, 2013). Other studies have demonstrated that, via "tools," an individual can achieve postural stability by using the haptic information of distal contact points (e.g., an older person who uses a cane to detect a surface that is suitable to maintain stable locomotion), whether with a fixed contact point (Jeka, 1997) or a mobile one (Albertsen, Temprado, \& Berton, 2010).

\section{An anchoring haptic model}

Experiments that include non-rigid tools help researchers better understand the role of haptic systems (Cabe, 2011; Cabe \& Hofman, 2012; Kinsella-Shaw \& Turvey, 1992). For example, Cabe and Hofman (2012) demonstrated that individuals are able to directly estimate the location of a target object (i.e., point of closest approach and bypass distance) by pulling on a string attached to a weight using their fingers. In a clever set of experiments, Kinsella-Shaw and Turvey (1992) found that individuals were able to perceive distances from their hands relative to occluded metal disks that were attached to a taut nylon strand line. By shaking the line, mechanical vibrations allowed the individuals to estimate the distance to the attached target object.

By combining the properties of non-rigid tools (such as the mechanical properties of strings ${ }^{2}$ ) that are connected to a target surface (Cabe, 2011; KinsellaShaw \& Turvey, 1992) and the well-known premise that body sway can be attenuated by touch (Holden et al., 1994; Jeka, 1997), we devised a system (i.e., the anchor system) that, for the purpose of postural stabilization, allows an individual to expand his or her references about the support surface by using a nonrigid tool. The system provides a type of anchorage - a string connected to a load with a given mass (a resistive probe), which remains in contact with a support surface (i.e., the floor). The individual's task is to pull the string with the hand just enough to feel the resistance of the load mass that is in contact with the surface, but without lifting it. By using the anchors, one in each hand, the individual has several options that are embedded within the task: ignore the anchors and not pull; increase the pulling forces (i.e., tensile forces) beyond the amount of resistance, hence lifting the loads off the floor; balance the pulling forces between both hands; or ignore one of

\footnotetext{
${ }^{2}$ Some mechanical properties of strings include density of the material, tensile strength, and elasticity. The primary mechanical properties of strings are (a) relatively resistant to tensile forces, (b) relatively non-resistant or compliant to compressive forces, and (c) relatively non-resistant to bending forces. Those are related to the properties listed, but for the purposes of mediating haptic information, tensile strength (typically, breaking strength) and elasticity (presumably very low) are secondary.
}

the anchors. The pulling can be continuous or not. In the experiments reviewed below, when participants violated the task instructions (i.e., pulling the loads off the floor), they were immediately asked to attempt to place the anchors back on the floor.

The anchor model (Figure 1) indicates that when the body, considered an inverted pendulum (i.e., in an unstable body position), is coupled with the adjacent flexible extensions (i.e., strings) of the anchors, the active handling of the strings expands the orientation reference with regard to the surface (i.e., perpendicular position). Figure 1 suggests that, from the center of mass $(\mathrm{CM})$ to the point at the base of the inverted triangle, when the $\mathrm{CM}$ moves away from the perimeter of support, the angle line of the vertical gravitational force component $\left(90^{\circ}\right.$ relative to the vertical) displaces from the (unstable) equilibrium point $\left(>90^{\circ}\right.$ relative to the vertical). The displaced angular distance to the unstable equilibrium point at which a person (or object) would fall over is determined as the "toppling point" (Cabe \& Pittenger, 1992). Farther from its equilibrium, the system needs more applied force to counterbalance the larger force values of the horizontal component. The magnitude of the required counterbalancing force depends both on the angular displacement and the weight of the object. Individuals, according to these authors, might operate with a time-to-topple tau, a haptic mechanism that is designed to help us explore unstable points of equilibrium. That is, applied to the illustration in Figure 1, in order to avert exceeding the topple point and preserve equilibrium, haptic "pushing" tau and "pulling" tau permit the system to control phase transitions or, in our case, avoid the loss of postural balance. The anchor system allows the individual to supply the minimum restoring force necessary to prevent the angular displacement to exceed the toppling point.

The tension on a flexible extension (i.e., the string) is affected by two sources of information: the resistance $(R)$ of the anchoring probes (loads) and the way in which an individual controls the extension itself by keeping the pulling forces $(F)$ below or equal to the resistance of the probes $(F \leq R)$. The anchoring probes are loads of specified mass, and the endpoints are connected to strings. In some experiments, the anchoring strings are attached to strain gauges that are fixed directly onto the floor. The act of controlling the extension also feeds back into the exploratory system, so that individuals are able to differentiate between changes in the tension caused by exploratory activity and changes in the body position (i.e., sway). In summary, motor control strategies that integrate haptic information collectively contribute to preserving postural stability.

Another aspect of the anchorage, as illustrated in Figure 1, is its potential to detect the distal spatial layout, serving as a reference frame for orientation and therefore expanding the body's perimeter of support relative to the surface. By integrating mechanical properties from the anchor system (string tension and mass resistance of the probes or loads), individuals 
Task A

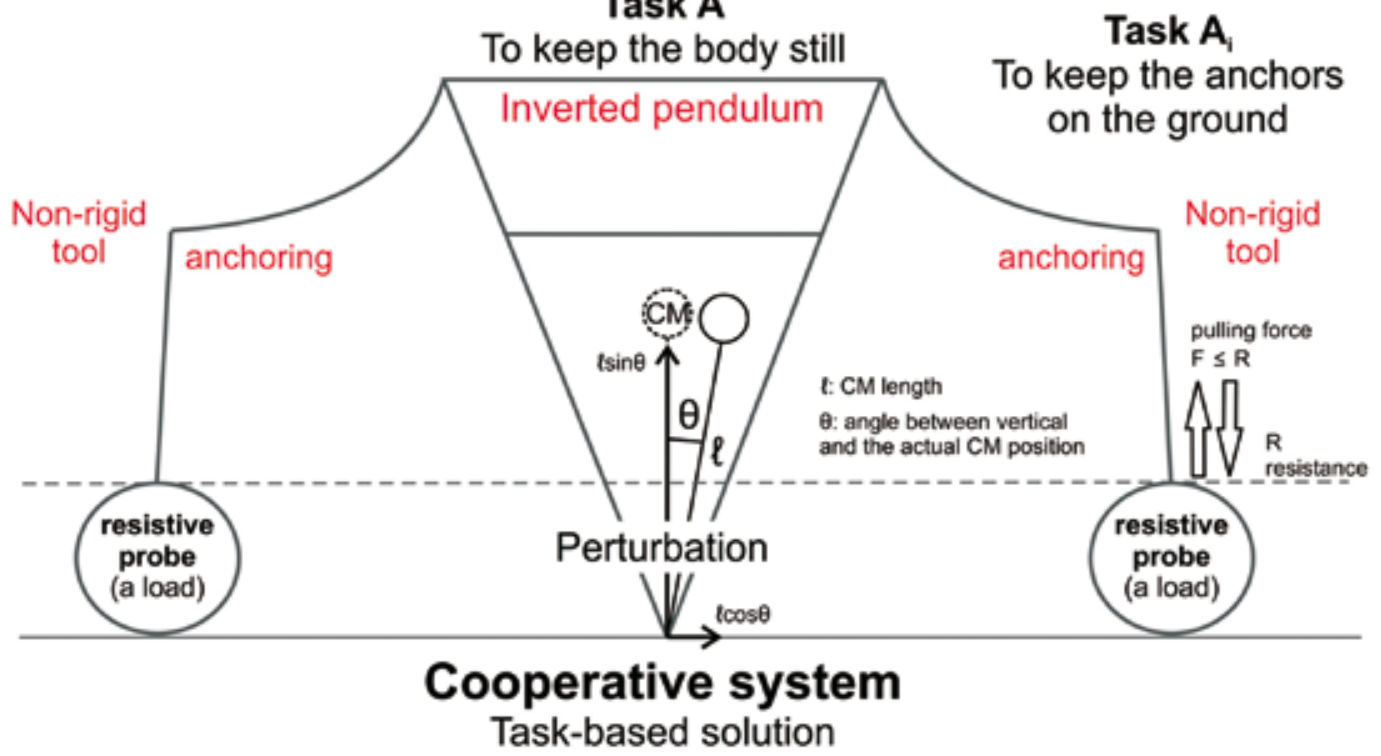

Figure 1. A schematic depiction of an anchor system model for a postural orientation task that uses haptic stimulus array information. CM, center of mass; $F$, force; $R$, resistance.

can use the haptic system as a perceptual telemodality (Cabe, 2013; Cabe \& Hofman, 2012; based on Von Fieandt's conception, published in 1966, "The world of perception"). Although haptic information is conveyed via the anchor system, ultimately it is useful information about the distal surface that is derived when these parts are integrated into an exploratory unit. In this case, the useful information in postural tasks results from the need for orientation of the entire body and its parts. Therefore, it is important to take into consideration that the hands, arms, head, torso, and legs all are elements involved in the anchor task.

While anchoring, the goal of the balance task is to maintain the body's center of mass (CM) within the limits of the base of support. The telemodality property of the anchor system expands the possibilities for individuals to explore the adjacent environment, ultimately resulting in increased postural stability. According to Cabe (2011), the term telemodality implies the ability of an individual to detect the properties of a distal object via the string-mediated connection.

During an anchoring task, the relationship between a (never quiet) postural system and a (deliberate, effortful, and dynamic) haptic information pick-up system becomes functionally tied because an ecological medium transmits energy patterns through various scale levels of the organism and its environment. The medium of anchoring is the physical connection between the hands and strings that integrates information about the surface that, in turn, is used to regulate posture. The flow of energy patterns through the strings (e.g., propagation and distribution of mechanical waves from subtle hand motion and resistance from the distal loads that rest on the floor) is (tele)transmitted via an architectural organization of the haptic system that relies on the relationship between the organism and the (anchor) tool.
Turvey and Fonseca (2014) claimed that the haptics' architectural organization-built from a net of muscles, connective tissue, and skeletal tissue, from the microto macro-level system scales - continuously receives and adjusts to information as a unit through an array of tensions. They offer a tensegrity hypothesis to explain why the non-uniform deformation of tissue is the basis for haptic perception. In the anchor system's case, whether deformation is transmitted within different levels of the organism's structural tissue/cell net or whether it is derived from non-biological connections with distal surfaces, in order to initiate, adjust, maintain, or terminate a particular task, a biological tensegrity must link the architecture (or structure) of the operating system to its (haptic) event process. Constituent elements of the body limbs that are connected to the anchor tool define the architecture of the operating system, and the anchoring task (designed to achieve postural stability) defines the event process.

\section{Anchoring for postural stability: our purpose}

Postural control, designed in part to keep the upright position, is one of the remarkable achievements of humans. Once developed, it ensures navigation and mobility skills that will enrich experiences throughout life. However, at any age, many impairments or disability conditions can affect balance (e.g., developmental disabilities, blindness, amputation, etc.). Furthermore, aging itself may cause balance deterioration that, in turn, becomes a risk factor for falls.

For a system to find an adaptive solution, it needs to be challenged. The basic natural challenge to the postural control system is gravity. However, as a subservient system to a complex net of motor behaviors, 
posture must be stable, although not immobile. There are situations, however, in which postural instability is too great and can affect adaptive behavior in general. To adapt, an individual who experiences deficits in balance must choose strategies to compensate for this difficulty. Protocols for intervention often include expanding or adding information to develop solutions and promote adaptation.

Therefore, because the anchor system paradigm can facilitate the development of postural control, as well as serve to rehabilitate, the technique can be considered an innovative low-technology tool to help solve reallife problems. Designed to haptically expand external references for orientation during postural tasks, the anchor system is an information-gathering tool that employs a non-competitive dual task (i.e., maneuvering the strings and maintaining balance). For an individual to use the anchor system for the purpose of maintaining balance, his or her postural control needs to be challenged. If a postural task is too easy, then an adaptive solution from this dual task would not likely be required. Also, the use of the anchor system could increase postural instability of the body because, while holding the strings, the hands and arms can also involuntarily move widely and abruptly, disrupting the body's alignment. For effective anchoring to take place, subtle movements of the hands need to contrast with the position of the anchor loads as a frame of reference relative to actual body position. However, if the postural state is disrupted to such an extreme level that an individual falls, then the use of the anchors to stabilize the body becomes an impossible task. In this case, arms and hands would involuntarily move due to stereotypical automatic reactions that are designed to rectify the body's posture, preventing the use of the anchors. Finally, intrinsic factors, such as motivation, attention to the task, comprehension of instructions, and efficacy of control strategies all are components of the anchoring process. Other (organismic) intrinsic constraints, such as the presence of a disability or immature developmental status, possibly would play a specific role in the way in which the anchor system is incorporated as an ecological niche (Figure 2). For instance, one of the task constraints of the anchor system consists of the goal of expanding the orientation's reference frame relative to a contact surface (e.g., the floor). However, such a constraint is tied to the intrinsic potential (organism constraint) of the user. If the attention factor is compromised, then an individual may not appropriately use the anchor strings for the purpose of achieving postural stability because he or she is distracted.

We adopt an anchor system model to demonstrate how individuals use non-rigid tools to dynamically process information from the support surface and adapt their behavior accordingly. In this article, we examine how the experimental findings of previous studies show that the stabilization of body sway can be achieved through haptic anchoring. Herein, anchoring is mediated by a non-rigid tool (a stretched string's tightening and loosening action, for example) connected to a resistive probe (i.e., a load mass) that is placed on a support surface (i.e., the floor). Our purpose is to discuss the results of a variety of experimental conditions that use an anchor system paradigm to illustrate the contribution of haptic perception to the postural control system. We aim to highlight several conceptual premises of dynamic system theory and the ecological perception-action approach and provide some insights into the potential application of this experimental paradigm.

We expanded the use of the anchor system in postural control situations to demonstrate how a non-rigid tool (i.e., a string) is used to mediate useful information from

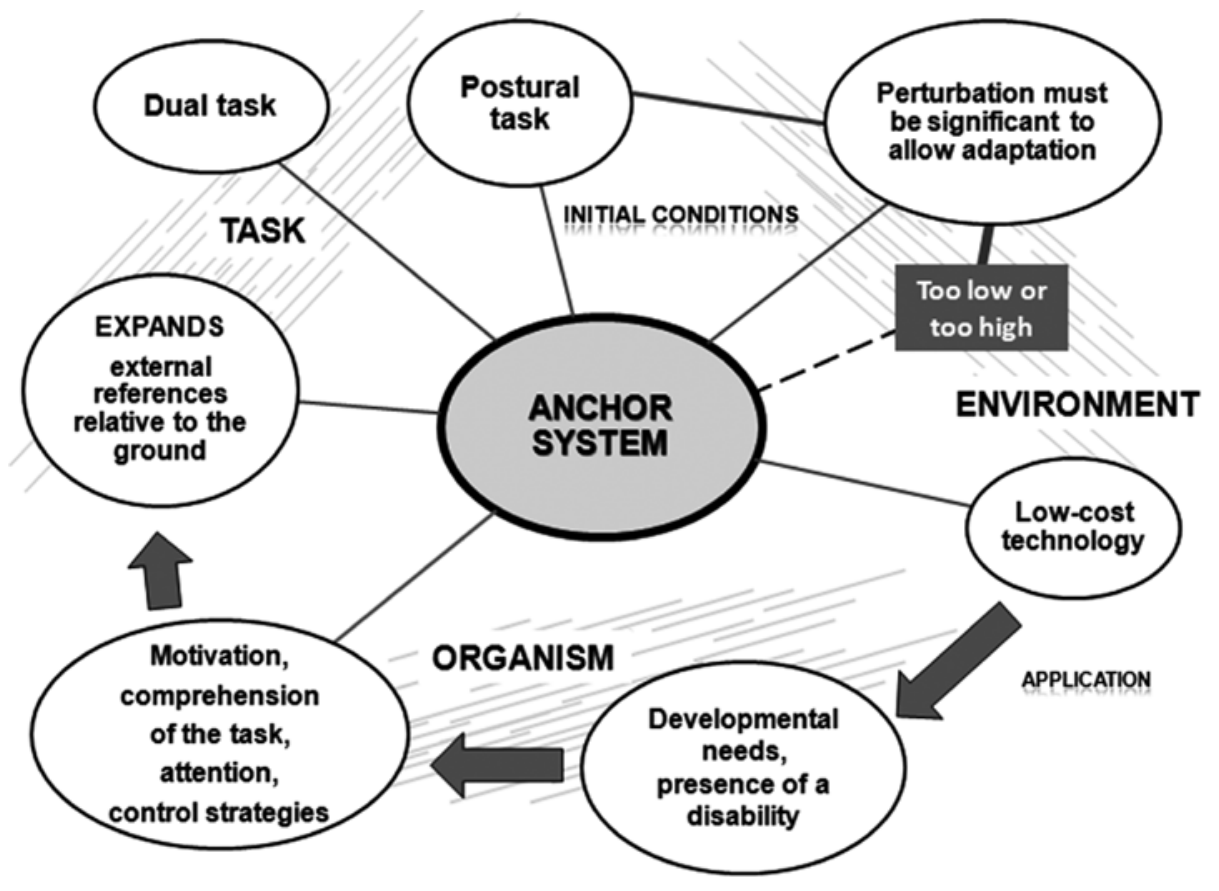

Figure 2. Context for behavioral adjustment using the anchor system. 
a moving support surface, such as during blindfolded walking. Additionally, we demonstrated how a dynamic interplay between less-stable anchor contact points also can provide individuals with useful haptic stimulus array information for postural stabilization (through a leash during dog walking, for example).

Here we present an overview of evidence from experimental tasks that used the anchor system and compare them to tasks that used the light-touch paradigm in order to highlight commonalities as well as the uniqueness of each of these two protocols. Finally, we highlight preliminary results from an intervention study that used the anchor system. That intervention was embedded in practical contexts of physical activity with the purpose of improving postural control in individuals with intellectual disabilities. The motivation for this study is the knowledge that this population typically experiences balance problems beginning at early ages. As these individuals get older, postural control deficits, especially in static balance tasks, continue to appear (Horvat, Croce, \& Zagrodnik, 2010). We also consider that, while handling the anchors is a voluntary action, high-function cognitive skills may not be a crucial element for the control strategies that are used in this task.

\section{Haptic anchoring as a "smart" perceptual system}

A non-rigid tool that mediates contact with a target surface and acts as a type of anchorage — one that provides stability to an individual-is much like an anchor that is attached to a boat that is floating in the water. The anchor load (or probe) and the anchor extension (e.g., a string or cable) provides stability to a system (an individual) so that postural orientation (e.g., balance) can be maintained in relation to an adjacent surface (e.g., the floor). If the loss of balance caused by perturbation affects the orientation, then individuals can anchor themselves by changing the tension on the strings just enough to feel the probes' (or anchors') resistance.

This metaphor was first proposed by MauerbergdeCastro (2004) when she identified robust effects of manipulating an anchor system during a blindfolded, unipodal task with young adults. In that first experiment, blindfolded participants held a pair of anchors while attempting to maintain a stable one-foot stance for $30 \mathrm{~s}$. Results of the comparison between a baseline condition (i.e., no anchors) and a condition in which the pair of anchors was used showed a significant reduction in the amount of sway. Sway was measured by the mean of moving window standard deviations of the kinematic data (i.e., angular position of the trunk segment). The individuals were able to use the haptic information via the anchor system to meet their postural stability needs. Furthermore and surprisingly, the magnitudes of the load masses (i.e., $1,000 \mathrm{~g}, 500 \mathrm{~g}, 250 \mathrm{~g}$, and 125 g) showed similar outcomes in this study. Therefore, variations in the anchor's properties, such as different mass loads, were not crucial in providing additional haptic information. A heavier load $(1,000 \mathrm{~g})$ would be expected to provide greater stability during loss of balance. However, the similar postural outcomes with the lightest anchor load (i.e., $125 \mathrm{~g}$ ) indicated that the individuals calibrated their pulling force just enough to provide the postural system with useful information to maintain stability (likely with pulling forces below the resistance of the $125 \mathrm{~g}$ load, or $\sim 1.22 \mathrm{~N}$ ).

That study was the first to test the anchoring paradigm and demonstrated that haptic anchoringdriven by necessity - led to adaptive solutions. That is, a successful behavioral outcome was the result of a "smart" perceptual mechanism that dynamically integrated information from the environment, the acting organism, and the dual task (i.e., anchoring and maintaining a still posture).

Although the haptic system is driven by (deliberate) exploratory activity, the interplay between anchoring and postural control is the result of a self-organized system and does not need an epistemic control process to calculate adjustments. During the haptic anchoring process, spontaneous postural solutions (adjustments) emerge from the continuous integration of local information that derives from evolving forces and their dimensions. These solutions push the system to adapt. Therefore, haptic anchoring is a "smart" perceptual system (Runeson, 1977) that self-organizes (posturalanchor-handling coupled system) behavior from local interactions between the components of an initially disordered (unstable posture) system.

\section{Experimental evidence of "smart" anchoring solutions}

Calve and Mauerberg-deCastro (2005) published the results of the anchor system's effects relative to postural stability in children who walked on a balance beam. Ninety children (30 each of 5-, 6-, and 7-yearolds) crossed a balance beam with and without the use of vision. The authors were interested in how developmental status influenced control strategies that employ haptic anchoring. Each age group was subdivided into three task subgroups (each with 10 participants), with separate subgroups that were randomly assigned to the following conditions: no anchors (control), $125 \mathrm{~g}$ anchors, and $500 \mathrm{~g}$ anchors. The results showed that, compared to the control group, the 7 -year-olds reduced their trunk range of motion in both anchor task conditions (i.e., $125 \mathrm{~g}$ and $500 \mathrm{~g}$ ). These children were able to maintain their walking stability by limiting the lateral sway of their trunk. The children in the 5- and 6-year-old groups did not efficiently use the anchor system and, therefore, did not differ from the group of children who performed the control task condition. The younger children appeared to be less attention-focused in such a task. Although no cognitive demand was required, attention appeared to play a role in the anchor's use for postural stabilization. 
In two other studies that used the anchor system, various levels of task difficulty ${ }^{3}$ in the postural task were tested in individuals with intellectual disability (Mauerberg-deCastro, Moraes, \& Campbell, 2012; Mauerberg-deCastro et al., 2010). The primary rationale for testing these individuals was to demonstrate whether or not the use of the anchors required higher levels of cognitive processing. Secondly, individuals with intellectual disability are known to experience deficits in postural control and in quality of movement (Shumway-Cook \& Woollacott, 1985). With this in mind, we expected that the presence of a disability condition would demand more efficient usage of the anchor system. In other words, the association between a disability condition and poor balance during an anchoring task would create demand for viable anchoring solutions to achieve postural stability.

Indeed, Mauerberg-deCastro et al. (2010) found that adults with intellectual disability, when blindfolded and performing a standing task (standing with both feet apart on a balance beam, with toes and heels not contacting the surface), recovered their balance more efficiently when they used the anchor system and when standing on a higher beam $(20 \mathrm{~cm}) v s$. a lower one $(10 \mathrm{~cm})$. Variability of the trunk angular peak velocity showed a significant reduction in the anchor condition when the participants used the anchors while standing on an elevated surface compared with the lower surface (Figure 3). The baseline condition showed that the higher beam appeared to be more challenging, since the significant statistical interaction between anchor task and height reinforced the evidence that participants who stood on the higher elevated surface likely better exploited the availability of the anchors. Based on other studies (Huffman, Horslen, Carpenter, \& Adkin, 2009; Pettersson, Olsson, \& Wahlund, 2007), we believe that the challenging tasks made these individuals more aware of the resources available because they were visibly preoccupied with a potential fall.

In a study with older participants (mean age, 70 years) who performed the postural task by

\footnotetext{
${ }^{3}$ In experiments that use the anchor system, the degree of postural task difficulty often raises safety issues, and many experimental restrictions can affect the routine of data collection. Because the majority of postural tasks are performed blindfolded, continuous monitoring of the degree of sway that may lead to falling is required. Familiarization with the task requires, besides positioning the feet and handling the anchors, stepping up and down from the testing surface to minimize fear and uncertainty about the surfaces' heights, typically stair steps. Additionally, experienced personnel are placed near the participants to ensure protection against potential falls. When sway is perceived to potentially lead to a fall (not a step down), the participant is physically held. In such a case, the trial is suspended and restarted after a brief pause. In the research protocol of our studies, especially when working with individuals with intellectual disability and older individuals, when a participant is unable to comply with the postural task and fails to complete two trials in a row (by falling or stepping down), he or she is dismissed from the study.
}

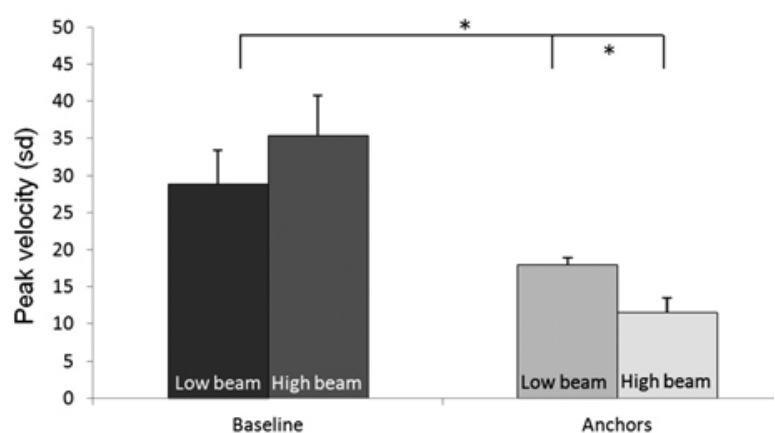

Figure 3. Variability of peak angular velocity (sd) of the trunk segment in the sagittal plane of individuals with intellectual disability $(n=11)$ who stood on a high $(20 \mathrm{~cm})$ balance beam (left) and low $(10 \mathrm{~cm})$ balance beam (right) in baseline and anchor test conditions. The lines above the bars depict standard errors. Unpublished chart using data previously published in Mauerberg-deCastro, E., Lucena, C.S., Cuba, B.W., Boni, R.C., Campbell, D.F., \& Moraes, R. (2010). Haptic stabilization of posture in adults with intellectual disabilities using a non-rigid tool. Adapted Physical Activity Quarterly, 27(3), 208-225. * $p \leq .05$.

maintaining a semi-tandem foot position with their eyes open, Moraes and Mauerberg-deCastro (2009) showed that both path length ${ }^{4}$ and mean sway velocity (anterior-posterior and medial-lateral directions) were reduced with use of the anchors. Although this task was relatively easier than the postural tasks that require blindfolded performance and challenging foot placement used in other studies by Mauerberg-deCastro and colleagues (Mauerberg-deCastro, 2004, $2010,2012)$, the aging factor can be considered an intrinsic constraint that challenges postural control. For the information mediated by the anchor system to be integrated into the postural task, the latter must be sufficiently challenging. That is, if the anchor-pulling task is not coupled with the postural task demand, then trials with the anchor system can have the same outcome as those without it.

Although these studies have shown a reduction in the amount of body sway and body sway velocity, we should consider the need for additional investigations using a different set of sway measures. Maurer and Peterka (2005) divided sway measures into three groups: displacement-, velocity-, and frequencyrelated measures. In our studies, we focused mainly on displacement-related measures and eventually velocityrelated measures. The inclusion of frequency-related measures as well as the nonlinear dynamical measures of the center of pressure (COP) signal (e.g., Donker, Roerdink, Greven, \& Beek, 2007) is essential to further understand the contribution of the anchor system to postural stability. At this point, the data that we have

${ }^{4}$ Path length is a parameter that is calculated as the sum of the center of pressure's displacement scalars in both the medial lateral and anterior posterior directions over a given task duration. A numeric increase in this variable is assumed to represent less stability, with a decrease representing more stability (Mauerberg-deCastro et al., 2012). 
allow us to claim that the anchor system reduces body sway and velocity in different populations and experimental paradigms. However, we are limited in our understanding of the inherent complexity of the postural control system with the addition of sensory information. Future studies should include a broader set of sway measures to characterize the effects of additional haptic information on postural control.

\section{Dynamic anchorage between coupled systems}

A recent study (Mauerberg-deCastro et al., 2013b) tested a dynamic anchorage while individuals assumed a variety of postural positions and at the same time held a leash attached to a dog that walked on a treadmill (Figure 4). In this task context, 10 blindfolded college students remained still for $30 \mathrm{~s}$ in a feet-tandem position. The control conditions included full vision and no dog. We used the mean COP path length as a measure of the amount of body sway. A significant interaction between vision and task indicated that the blindfolded individuals who held the dog's leash better controlled their balance than those who performed the same task without the dog (Figure 5). The full-vision condition resulted in similar postural performance in both task conditions (i.e., no dog and dog). The dynamic activity of dog walking contributed to the postural orientation of the handler only when challenges were sufficient to significantly deteriorate postural behavior during vision deprivation.

Similar results were also found in a study with college-age participants (Périco et al., 2013) and in a study with adults with intellectual disability (Mauerberg-deCastro et al., 2013c). In both those studies, the participants walked on a balance beam while simultaneously walking a dog on a leash. When the individuals were deprived of vision, the walking of the dog positively reduced the variability of the kinematic parameters of walking in both groups. In these studies, when the participants walked the dog,

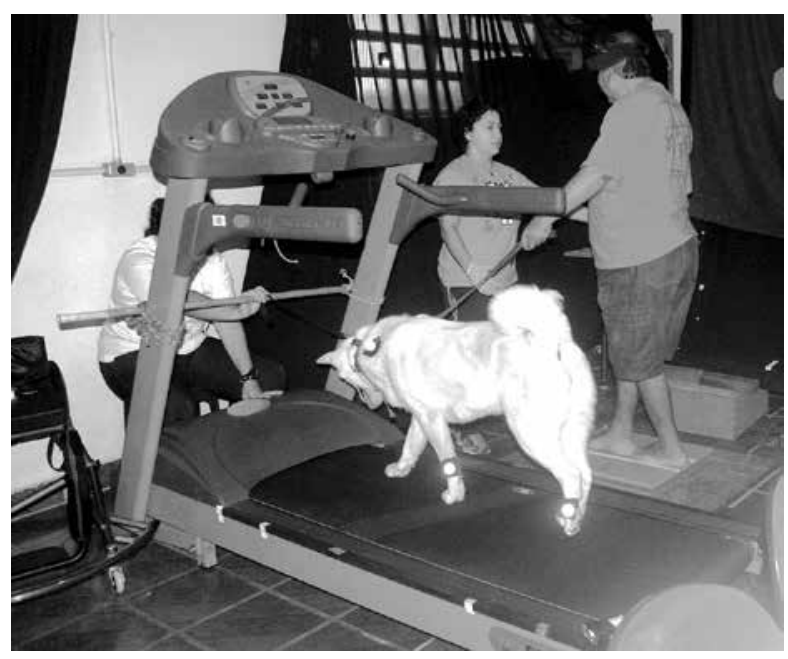

Figure 4. Illustration of dynamic anchorage in which an individual performs a postural task and simultaneously holds a leash attached to a dog that walks on a treadmill.

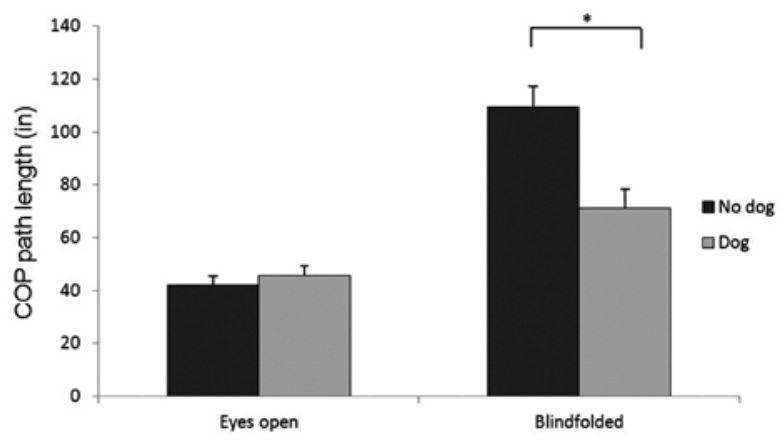

Figure 5. Mean values of the path length of the center of pressure (COP) of participants $(n=10)$ who performed a balance task with eyes open and blindfolded while simultaneously walking a dog and in a condition with no dog. The lines above the bars depict standard errors. ${ }^{*} p \leq .05$.

all of the kinematic parameters (i.e., duration of each stepping cycle, inter-limb relative phase, duration of double support phase, swing phase, and linear velocity of stepping) showed significantly reduced variability (measured as the standard deviation of walking steps or stepping cycles). Therefore, the use of haptic information relied on functional demands created by task constraints (i.e., walking blindfolded with the dog).

The dog's walking dynamics contributed to the postural orientation of the handler only when task constraints were sufficiently disruptive to significantly deteriorate postural behavior (as with vision deprivation). Anchorage between the handler and the dog provided haptic information that was useful to the handler's orientation ${ }^{5}$. From a human behavioral perspective, the less stable (and less predictable) source of haptic input (i.e., the dog) acted, via the dog's leash, as a dynamic spatial reference for orientation to the postural system. On the other hand, when vision was available to the human, handling the dog became a separate task from the standing task; therefore, haptic information for the postural control system was largely

${ }^{5}$ In this experiment, a female dog was trained to walk on a treadmill and respond to leash commands (e.g., keep walking and maintain a straightforward direction). The individuals were instructed about the leash commands and were briefly trained on how to control the dog. They were instructed to keep the leash relatively tight but not so tight as to pull the dog off the treadmill. The individuals were able to perceive the paced rhythm of the dog's walking and were encouraged to use the dog to help them maintain postural control. During an experimental trial, sometimes the dog's walking direction deviated on the treadmill belt, causing jerky pulls on the leash and potentially threatening the human's postural control. In order to minimize extreme situations of the dog's noncooperation, a second leash was available to an assistant who was positioned in front of the dog. This was used as a security measure so that the dog would not be able to leave the treadmill, as it could cause the human to fall in the blindfolded task conditions. Additionally, there were rare instances when the human individual lost balance, producing jerky movements through the leash that affected the dog's task. In such cases, the trial was reinitiated. 
irrelevant. The haptic information was embedded in a crude and sometimes abrupt exchange of postural influences through the leash. At times, when using the leash to regain control of the dog's walking or its position on the treadmill, haptic contact may not be subtle. Even subtle destabilizing events that are caused by the dog's walking motion on the treadmill are felt through the pulled leash. Yet, in spite of such motions, individuals when blindfolded were able to haptically use the leash to control the dog's position on the treadmill and also create a reference frame to stabilize their postures. The human detects the dog's walking rhythm through the leash, which also informs the human about his or her relative position. The voluntary management of the dog's walking provides an intrinsic dynamic flow from an action-perception cycle. The stochastic, yet not deterministic, nature of the dog's movements (i.e., not completely predictable moment-to-moment, but neither completely random), provides, firstly, a context for perturbation and adaptation that continuously affects the levels of stability of the human posture and then, a means for controlling the dog's movement because the leash is a two-way "conduit" of information.

Managing the dog's behavior through the leash is a supra-postural activity task that reflects an anchoring phenomenon. Therefore, to control the dog, the postural oscillation of the handler may need to be minimized to perform this task. Through anchoring, the dog's behavior becomes a subsystem to the human's postural control system. From the dog's perspective, subtle pulling commands through the leash likely help the dog maintain its walking at a paced rhythm. As we said earlier, although the treadmill belt imposed regularity on the dog's walking cadence, the dog could modify its step length, direction, and orientation on the treadmill surface. Therefore, because this dog maintained the walking treadmill task requirement, haptic information evidently was available through the leash. The walking task carried out by the dog and the postural task carried out by the human handler were softly interconnected by the non-biological extension, therefore, demonstrating how a tension array relationship (i.e., a tensegrity phenomenon by Turvey and Fonseca, 2014) of an haptic mechanism resulted in a behavioral solution (e.g., postural stability).

\section{Anchorage: haptically detecting changing force levels}

According to Rabin et al. (2006), when individuals employ light touch on a surface with a fingertip to avoid leaning during a postural task, they can use a force level as low as $\sim 0.4 \mathrm{~N}$. Additionally, they claim, "...such a force level cannot attenuate postural sway by providing mechanical stability" (p. 122). In the experiment by Rabin and colleagues', individuals stood on a force platform for $25 \mathrm{~s}$ while lightly touching a surface mounted on strain gauges. According to these researchers, the average fingertip force during the initial contact was $0.79 \mathrm{~N}$.
Throughout the task duration, the force decayed to 0.46 $\mathrm{N}$. In that experiment, the participants were not allowed to use force levels above $1 \mathrm{~N}$.

To identify whether or not subtle contact with a nonrigid extension tool is relevant for orientation purposes in the anchor task, we tested the extent of pulling force levels in a balance task using the anchor system (manuscript in preparation). Each of 12 blindfolded adults, while maintaining a feet-parallel position on a balance beam for $30 \mathrm{~s}$, held a pair of anchor strings that were attached to strain gauges (one dimension vector) that were fixed to the floor. In this foot position, the participants kept their foot arches in contact with the surface while heels and toes did not touch the surface. This position is quite difficult to maintain because, when heels and toes do not contact a surface, they limit ankle control strategies and affect the postural control. Their instructions were to gently pull the anchor strings, but not at the expense of abandoning the balance task. Therefore, when their instability increased, they would have tried to counteract it by using the anchors.

In this study, the mean pulling force levels on the anchor system were $2.1 \mathrm{~N}$ and $2 \mathrm{~N}$, respectively, for the right and left hands. Peak force events were twice that amount (i.e., $4.7 \mathrm{~N}$ and $4.6 \mathrm{~N}$ respectively, for right and left hands). Although the variability of pulling force levels was high, both hands tended to work in synchrony. In Figure 6, the patterns of pulling forces of both hands of three participants (TAC, NAT, and ACA) show that these participants used both hands predominantly in-phase throughout the 30-s duration of the trial. However, we also noted various time segments in which the hands' pulling forces (see participant ELI) did not show an apparent coordination pattern. The assessment of coordination patterns (i.e., in- and outof-phase patterns), however, would be possible through a more specific signal processing analysis, such as through the use of relative phase angles.

When participants pulled the strings attached to strain gauge cells fixed on the floor, they haptically used the resulting resistance as information to achieve postural stability. In previous experiments, the participants were instructed to keep the anchor loads on the floor because they were not fixed on the surface (e.g., MauerbergdeCastro, 2004). The pulling forces were not measured in this latter task context.

Handling the anchor strings can involve a large number of degrees of freedom with the movement of the hands, wrists, and other distal joints. The variation on pulling forces is derived from two processes. One is related to the entrainment between the hands and body that produces the pattern of the body postural sway, mirroring the hands' pulling pattern. The other involves the potential for these upper body segments to decouple from postural sway in order to free the hands and fingers for haptic exploration to attenuate postural oscillation upon demand (i.e., when instability becomes critical). The data that we have so far do not allow us to differentiate between these two processes. To do 

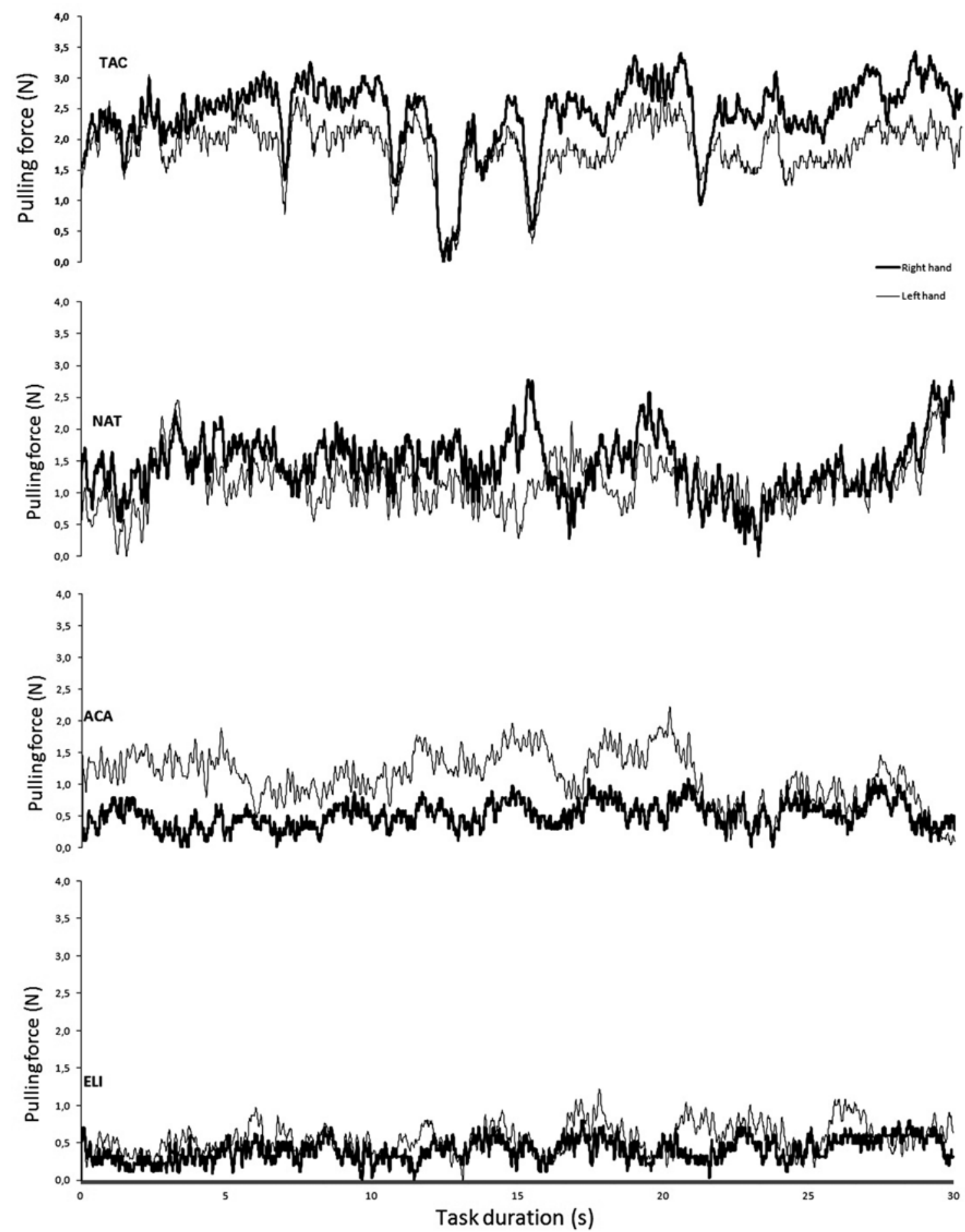

Figure 6. Time series that depicts in- and out-of-phase pulling forces $(\mathrm{N})$ between hands while pulling the anchor system during a single 30 -s task trial of four participants.

so, it would have been necessary for us to measure the horizontal forces applied on the anchors to crosscorrelate with the COP displacement, as originally done by Jeka and Lackner (1994). In that case, we would expect that the highest correlation would occur with a time lag, meaning that changes in force applied to the cables would lead to relative changes in the COP trajectory. Under those conditions, the coupling process would predominate. Another option would be to run a coherence analysis based on the power spectrum density of both horizontal cable force and COP trajectories. Coherence close to 1.0 would indicate that both signals are coupled, suggesting that the first process would also predominate. Future studies should address this issue.

Another study by Mauerberg-deCastro et al. (2013a) compared strategies of haptic contact during postural tasks under the two paradigms, the light touch and the anchor system. The comparison between the two experimental protocols allowed us to examine particularities and commonalities of these tasks in 
order to demonstrate the role of haptics. For instance, both protocols imply that only light or subtle contact is needed for the detection of information. In both, the behavioral outcomes result in the stabilization of posture. During the light touch task, individuals make light contact downward, directly with the surface, which creates a reference for postural orientation. In the anchor task, mediated by a soft tool, the upward contact from the surface - via the string - provides a reference for postural orientation. The use of the anchors requires individuals to make subtle exploratory motions with their hands. Hand motions are restricted by the task conditions (i.e., keeping the strings taut while the load rests on the floor's surface).

In order to compare light contact with anchoring, 20 blindfolded adults stood for $30 \mathrm{~s}$ with their feet in a tandem position on a force platform. In the light touch task, the participants moved their index fingers downward to lightly touch a surface (a strain gauge pad, one dimension vector). In the anchoring task, the participants held in each hand a flexible cable (stretched taut) that was attached to a strain gauge pad that maintained contact with the floor (anchor system). The contact surface for the light touch experiment was located in front of the participants. A non-contact condition was used as a control. In contrast to the control condition (65 in), the amount of body sway measured by the center of pressure's path length significantly decreased during performance of the anchor task condition (46 in), as well as during the light touch condition (27 in).

The study's second question referred to the amount of force on the strain gauges in each task: pulling or tensile force for anchor and compressive force for light touch. In the anchor-task condition, the participants typically employed twice as much force than in the light touch task condition, with the maximum pulling force almost five times higher than the downward forces employed in the light touch task context. The amount of pulling force in our study was similar to values found by Rabin et al. (2006) in the light touch tasks in which the average fingertip force during the initial contact was $0.79 \mathrm{~N}$. In our light touch and anchor tasks, the mean force was $0.7 \mathrm{~N}$ and $1.6 \mathrm{~N}$, respectively.

Although Rabin et al. (2006) claimed that force levels above $1 \mathrm{~N}$ could provide mechanical support for the stabilization of posture, the balance task demands in our anchor study are different from the demands of the light-touch design. As noted earlier, the anchor system is a multi-degree of freedom tool that allows the hands and arms to distally move around the point of contact on the surface via the flexible cord. All of these factors make the anchor task different in important respects and, perhaps, not entirely comparable to the light touch task, with the exception that both employ haptic stimulus array information for the purpose of postural stabilization.

The anchoring task has potential for practical applications in everyday locomotion activities, exercise, and motor activities, given the unique way in which individuals handle the anchor system to haptically orient the body's position in space. The pulling motion during anchoring is possible when individuals drag the anchor loads (either with one or two hands) during the walking tasks performed on different surface layouts (e.g., balance beams, ramps, through apertures, and so forth) or simply during a standing balance task while they simultaneously call upon another motor skill (e.g., standing on one foot while kicking a ball or standing on a balance board while throwing a ball toward a target). The pulling forces are determined by the load mass. If the load is lifted from the contact surface, then the anchoring task no longer exists.

A light touch task context, although requiring specific instructions not to exceed a predetermined amount of pushing force, does not prevent an individual from leaning the body's weight on the surface for the purpose of balancing. This is like an individual who uses a cane to support his or her body weight to prevent loss of balance. The anchor task context is not compatible with leaning (one cannot lean on strings). It requires participants to pull the loads just slightly while at the same time, keeping the loads in constant contact with the surface.

As a candidate tool for intervention purposes, the anchor tool could help participants improve balance and the strength of the muscles involved in independent upright positions and locomotion. Many balance therapeutic protocols encourage exclusive reliance on mechanical support, such as the use of walker devices, for example. Anchoring requires full control of the lower limbs and release of the upper limbs for subtle exploration of the anchors. Therefore, anchoring tasks in therapeutic protocols or embedded in exercise programs could help an individual preserve the control of balance and recover the strength of the lower limbs.

\section{Are there practical applications for an anchor paradigm?}

One potential application of an anchoring tool is for aiding groups who have balance problems. For example, as individuals get older and become less active, they show a higher risk of falling because of balance problems. Using the anchor system paradigm, Dascal, Okazaki, and Mauerberg-deCastro (2012) compared the performance of older adults (mean age: 68 years) and young adults (mean age: 20 years) in a postural task that used a semi-tandem foot position. The conditions included full vision and occluded vision during use of the anchor system $(125 \mathrm{~g})$. A baseline condition without the anchor system was used for comparisons. The dispersion, amplitude, and velocity of sway of the COP medial-lateral and anterior-posterior directions showed that both older and young adults exhibited a reduction of body sway when using the anchor system, with one notable difference. For the young adults, relative to the baseline condition, the effectiveness of using the anchor system was better in conditions in which the visual 
information was absent. For the older adults, relative to the baseline condition, the usefulness of the anchor system was superior in conditions in which the visual information was present (Dascal et al., 2012).

In another study with adults with and without intellectual disability, Mauerberg-deCastro et al. (2012) again found that the anchor system was efficient as an aid for postural tasks, reducing the path length of the COP when comparing pre- and post-practice without the anchors. The adults with and without disabilities improved their postural performance equally when using the anchors, in contrast to the pre-practice trial. These results reinforce the potential short-term adaptation learning effect after continuous use of the anchor system.

Freitas, Mauerberg-deCastro, and Moraes (2013) questioned whether the continuous use of the anchor system improves postural control after its removal. They evaluated the effects of practicing balance tasks using the anchor system on postural control in healthy older individuals. The practice protocol included trials of standing posture in the semi-tandem position with full vision available. They allocated participants into three groups: $0 \%, 50 \%$, and $100 \%$. These values corresponded to the percentage of trials that each group used the anchor system during the practice protocol. The $0 \%$ group never used the anchor system during the practice protocol, whereas the $50 \%$ group used the anchor in half of the trials of the practice protocol, and the $100 \%$ group used the anchor system in all of the trials of the practice protocol. A pre-practice phase (without anchor) was followed by a practice phase (with the anchor system at the predefined frequency) and a post-practice phase (immediately after and late after without anchor). The practice protocol occurred on two separate days, with an interval of $24 \mathrm{~h}$. Fifteen minutes after finishing the second session, participants performed the immediate post-practice phase. Twenty-four hours after finishing the second session, participants performed the late postpractice phase.

The older adults' postural control improved after they practiced the standing postural task, irrespective of the group. All three groups showed a persistent effect (i.e., the amount of body sway reduced compared to the pre-practice) $15 \mathrm{~min}$ (immediately) after the end of the practice phase. Surprisingly, however, $24 \mathrm{~h}$ after finishing the practice phase, the group that used the anchor system $50 \%$ of the time maintained their postural control gains, whereas the other two groups returned to their baseline (pre-practice) values. In the practice protocol with $50 \%$ frequency of anchor use, the contrast between trials with and without the anchor system characterized a type of task variability that likely led the postural system to adapt. Such contrast in the task requirement suggests that the haptic information helped the participants recalibrate the sensory integration process in the absence of the anchor system (during the late post-practice phase).

In another study that tested effects of practice (Mauerberg-deCastro et al., 2012), the systematic use of the anchor system affected the postural control system by reducing body sway after short-term practice in both adults with intellectual disability and normal adults. Two control groups (i.e., individuals with intellectual disability and normal adults) who performed the same number of trials without the anchor system (i.e., control training) showed no improvement in postural performance. The individuals with intellectual disability and the normal adults who performed three blocks of trials using the anchor system showed a rapid reduction of the amount of sway (i.e., path length of the COP) during the conditions with the anchor (Figure 7). Most importantly, the final baseline for both groups of individuals who used the anchor system showed a significant improvement relative to the initial baseline. These results illustrate how haptic information gathered via the anchor system can, in a brief amount of time, help individuals reorganize their ability to control their posture. The removal of the anchor system resulted in better performance relative to the initial trial. This was contrary to a study by Freitas et al. (2013), who did not confirm these findings for older adults. Their results showed that performing the balance task (i.e., baseline task without the anchor system) immediately after the blocks of practice that used the anchor system resulted in no effect in their postural control parameters; however an improvement appeared $24 \mathrm{~h}$ later. We suspect that the level of difficulty of the balance task imposed fewer demands on the postural system; therefore, the use of the anchor system limited its value for short-term adaptation.

The encouraging results with regard to potential applications of the anchor system in rehabilitation settings was further tested by Polanczyk (2003), and MauerbergdeCastro and colleagues (Mauerberg-deCastro, Calve, Viveiros, Polanczyk, \& Cozzani, 2003; MauerbergdeCastro et al., 2013b; Moraes \& Mauerberg-deCastro, 2009). They determined that the portability of the anchor system, its low-cost technology, and the diversity of balance activities into which the anchor system could be incorporated would be beneficial in the applied field of rehabilitation. The rehabilitation of individuals with intellectual disability starts very early in life and tends to continue for many years throughout adulthood. Sometimes, prescription of rehabilitation protocols is continuous because aging brings new challenges (Uyanik \& Kayihan, 2010).

Polanczyk (2003) subjected a group of individuals with intellectual disabilities to a 1-month intervention physical activity program that used the anchor system as an aid in postural tasks, both in dynamic and static contexts. Figure 8 illustrates activities of the training sessions that used the anchor system. Pre- and posttests included standing blindfolded on a balance beam while maintaining a parallel-feet position for $30 \mathrm{~s}$. The percentage of time that the participants stayed on the beam increased after the intervention. During the pretest, these participants needed mechanical aid from an assistant to prevent them from stepping off the balance 

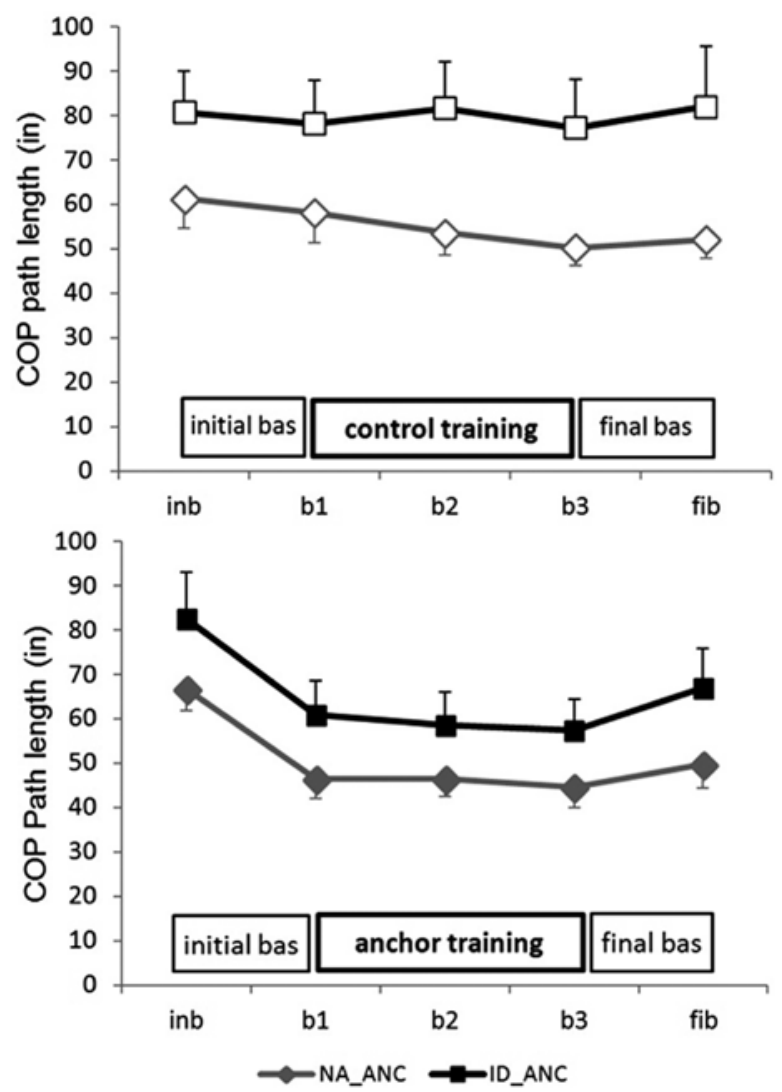

Figure 7. Mean values of the path length of the center of pressure in groups with intellectual disability (ID) and normal adults (NA) across blocks of practice (b1, b2, and b3) and baseline (bas; initial and final baseline [INB and FIB], respectively) during the postural task. Two independent groups represent, respectively, the control training experimental setting (CON) (top) and anchor training experimental setting (ANC) (bottom). The vertical lines depict standard errors. Adapted chart using data previously published in Mauerberg-deCastro, E., Moraes, R., \& Campbell, D.F. (2012). Short-term effects of the use of non-rigid tools for postural control by adults with intellectual disabilities. Motor Control, 16(2), 131-143. * $p \leq .05$. beam. After intervention, the individuals were able to stand significantly longer on the balance beam by themselves, particularly in the baseline condition tasks, which they performed without the anchors.

More recently, a longitudinal study that used the anchor paradigm was initiated by Mauerberg-deCastro and colleagues (Mauerberg-deCastro et al., 2013b). During a school semester, 12 adults with mild and moderate intellectual disability attended 24 sessions in which the anchor intervention protocol was used. A year later, a control group also was subjected to the same adapted physical activity protocol but without the anchor system. In the laboratory, postural tasks were employed pre- and postintervention. For $30 \mathrm{~s}$, blindfolded individuals maintained a parallel bipedal position on a balance beam that was placed on a force platform. Trials were conducted with and without the anchor system. COP path length was obtained for two directions of motion: medial-lateral and anteriorposterior. After the period of intervention, the experimental group exhibited a significant reduction of body sway. The postural tasks that were performed without the anchor system showed the most significant improvement after intervention compared with the tasks that used the anchor. The intervention affected performance in all of the task conditions that were performed in the laboratory, but especially in the condition that did not rely on haptic perception (i.e., baseline). The control group, although also significantly better at balancing when using the anchor system, did not significantly reduce their path length after the intervention program. The preliminary results of this longitudinal study (in progress) show that individuals with intellectual disabilities can detect the stabilizing properties of the anchor system haptically in order to regulate their posture. Additionally, the anchor system shows potential usefulness as a therapeutic tool that can be integrated into adapted physical activities (see Figure 9).

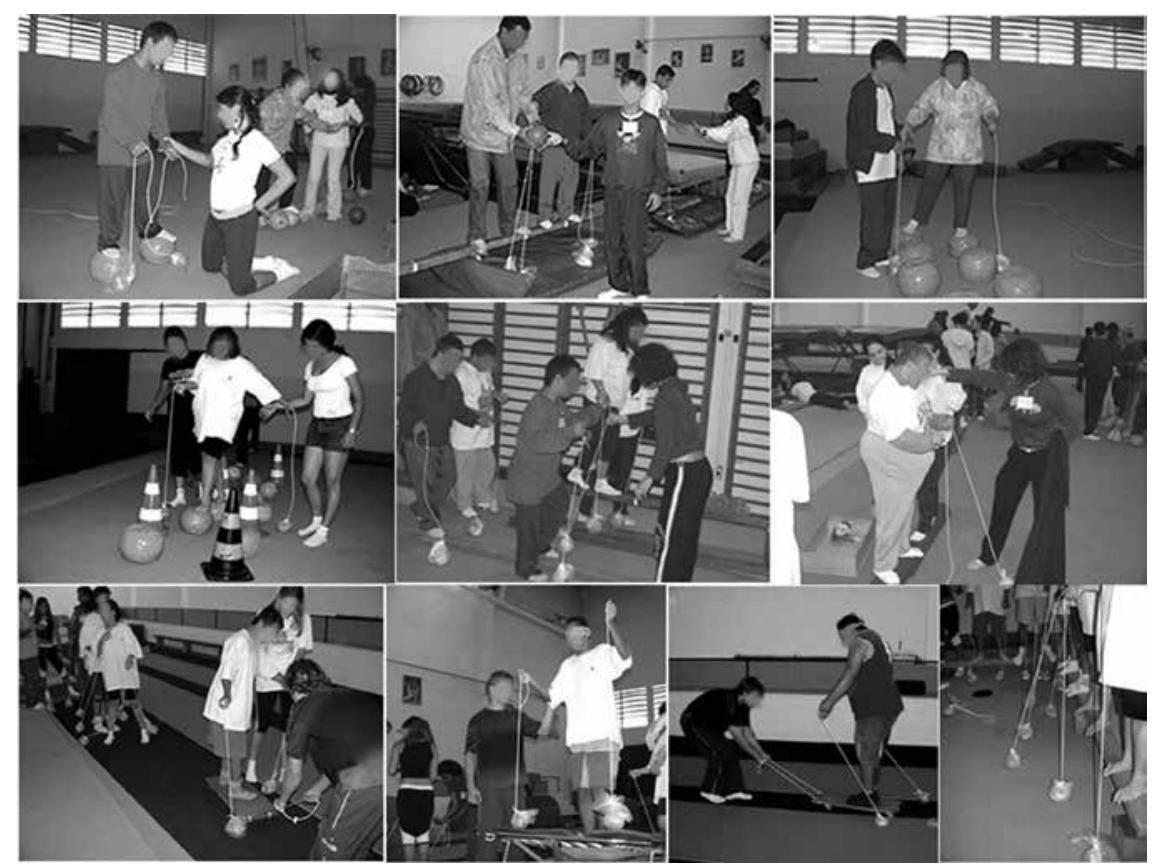

Figure 8. Examples of balance tasks during intervention sessions that used the anchor system in individuals with intellectual disabilities. 


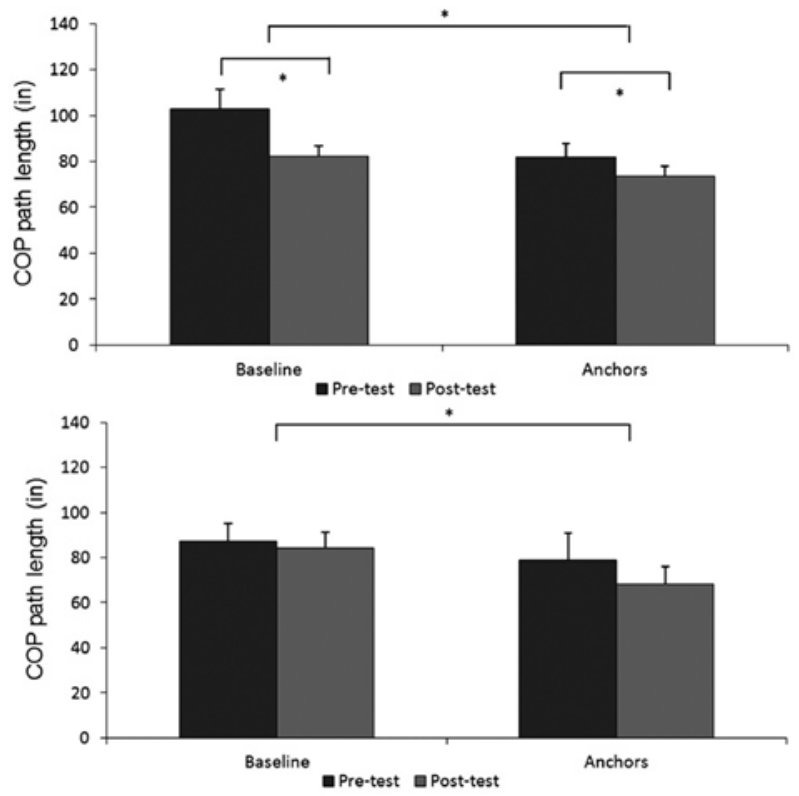

Figure 9. Mean values of the path length of the center of pressure (COP) of in groups with intellectual disability who were subjected to the experimental anchor setting (top) and control training (bottom). The lines above the bars depict standard errors. ${ }^{*} p \leq .05$.

One puzzling question involving these rapid adaptive behaviors (i.e., achieving stability via haptic anchoring) is how functions (e.g., the control of posture) of individuals with disabilities or individuals who are undergoing the degenerative process of aging are optimized in such a short term. One approach is to analyze the intrinsic changes that take place at the neuronal level because intellectual disability, for example, is attributable to extensive and diffuse insult to the brain (as well as to other parts of the organism, such as is commonly observed in Down syndrome).

Wagman, Shockley, Riley, and Turvey (2001) observed that fast haptic perceptual learning occurs when an individual fine-tunes his or her behavior to attend to the task-relevant variables, calibrate the task spatial scales, and explore task-changing relevant parameters. For these authors, such a fast learning process involves more complex mechanisms than those observed in neural network models that are responsible for slow perceptual learning and that lead to long-term adaptations and structural modifications.

For Petrov, Dosher, and Lu (2005), as a result of practice, perceptual learning improves performance because the system selectively and distinctively weights the relevant network model to be in tune with task constraints. According to these authors, the outcome performance or behavior derives from connections that are anchored in a stimulus-specific unit but can also account for learning transfer. Although their experimental tasks required representations and decision structures to account for response accuracy, our haptic anchoring task also requires fine tuning of the anchors' handling of the task demands for the achievement of postural stability. Accuracy in such handling activity is ultimately a consequence of weighting information relevance in order to maintain an upright position. Such weighting may reflect neural plasticity.

Functional and structural plasticity often helps brain construction and storage repair and is the result of both genetic mechanisms and experiences that accrue over one's lifetime (Vaillend, Poirier, \& Laroche, 2008). Functional plasticity occurs in different time frames, often on broad scales. Additionally, the known plasticity of neural networks (Fernandez \& Garner, 2007) can be influenced by the plasticity of non-neuronal elements, such as astrocytes, oligodendrocytes, and vasculature (Vaillend et al., 2008). According to these authors, these non-neuronal elements seem to account for immediate changes in the microenvironment of synapses, such as changes in blood flow.

As experience and learning modulate gliogenesis and the growth of astrocytes (Molofsky et al., 2012), the learning component of the anchor task could be an opportunity for non-neural plasticity. Non-neuronal plasticity would, at different times, modify neuronal plasticity through the integration of information patterns in diverse structures (e.g., tuning somatosensory input skin patterns and kinesthetic inputs), which in turn would add to the explanation of why haptic anchoring modifies postural orientation. A physiologically relevant level of activity derives from a dynamic interplay of collective compensatory mechanisms that are observed in an acting organism (Fernandez \& Garner, 2007). Anchoring of the posture provides a significant experience in which these mechanisms may evolve. Some mechanisms determine how internal or external inputs modulate, update, and integrate a number of motor structures, such as when the primary motor cortex outputs to spinal cord motor neurons and then to the trunk and limb muscles (Tibbetts, 2004). Others modulate the chronic excitation or inhibition of neuronal networks (Fernandez \& Garner, 2007). These mechanisms evolve on the basis of shortand long-term experiences provided to the organism.

The potential long-term changes that are affected by such experiences (e.g., haptic anchoring) rely on an ongoing functional and nonfunctional plasticity process that is affected by intrinsic factors (e.g., maturation and genetic potential) and extrinsic factors (e.g., rehabilitation stimulation or impoverishment of stimulation). Therefore, ongoing experiences continue to promote the organism's plasticity, whether it is functional or not.

Chechlacz and Gleeson (2003) claimed that the literature supports the view that intellectual disability results from alterations in molecular pathways that underlie neuronal processes that are involved in cognitive functions and other behavioral functions. For these authors, insults to the brain that cause developmental disability affect structures in a relatively fixed way (e.g., neuronal cell loss, stunted dendritic branching, and spine dysgenesis), and chronic changes in central nervous system activity that arise over time can directly modify the cellular composition of a neural circuit. Yet, individuals 
with intellectual disability have diverse experiences and engage in an intrinsically motivated search for stimulation that may warrant potential short-term adaptations.

Long-term benefits after terminating the use of the anchor system might be offset or attenuated due to the organism's (body's) changes towards dysfunctional plasticity (e.g., aging). A lifetime condition such as an intellectual disability has the potential to maintain ongoing dysfunctional plasticity. As with the case of aging, the degenerative process associated with balance problems will likely prove, over time, to be a losing battle. Rehabilitation protocols and personal active lifestyles that help slow the degenerative process (although not indefinitely, given our mortality) are contexts that force functional plasticity.

\section{Concluding remarks}

The science of haptics has provided us with knowledge about adaptability that has contributed to numerous applications: in the field of prosthetics or haptic technology (Kim, Colgate, Santos-Munné, Makhlin, \& Peshkin, 2009); rehabilitation techniques for the integration of sensory feedback after amputation (Chatterjee, Chaubey, Martin, \& Thakor, 2008); and haptic feedback in robot-assisted surgery (Okamura, 2009). The science of haptics has increasingly expanded to the development of interface technology in teleoperations in microgravity (Ando, Ohta, \& Hashimoto, 2000). Also, it has provided a better understanding of haptic cognition in astronauts, including situational awareness, modeling cognitive workloads, and evaluating the usability and effectiveness of human-automation interface exposure (National Research Council, 1997). Additionally, various methods of rehabilitation (e.g., multimodal integration therapy, neurodevelopmental techniques, and the Feldenkrais method) can provide insights about haptic functioning (Uyanik \& Kayihan, 2010).

Studies that demonstrate robust effects of haptic contact on postural stability, such as light touch and, more recently, those that use the anchor system, are quite numerous. Since its inception in 2004 (MauerbergdeCastro, 2004), various populations have been assessed and results have been replicated, as summarized in this article. The interactive relationship between an individual and the anchor system tool constitutes an action-perception cycle.

Direct or indirect contact, as well as static or moving distal sources of contact are known to increase the perception specificity that arises from the task array. Based on Petrov et al. (2005), we propose that anchoring can help the perceptual haptic system selectively and distinctively weight the relevant variables from a set of task and environment (e.g., surfaces, objects, substances, and events) constraints. For example, during the anchorage established through a leash attached to a moving dog, perceptual weighting requires fine tuning and eliminating redundancies from the task array. The dog has certain degrees of freedom to move on the treadmill surface to ultimately provide the human handler with a framework that is suitable for the maintenance of an upright posture or controlled walking (MauerbergdeCastro, Melo, \& Périco, 2011; Mauerberg-deCastro et al., 2013b, c; Périco et al., 2013). Weighting relevancy also requires fine tuning to the priority demands of a task context, such as those observed during varied task challenge levels. Anchoring to a postural task is more helpful when the difficulty level is higher than when it is lower. In challenging postural tasks, individuals pay more attention than during less challenging ones (Mauerberg-deCastro et al., 2010).

The anchoring process is a dynamic system of components that operate in concert to help maintain an upright and stable body position, utilizing automatic postural responses and intentional control mechanisms during haptic exploration. Such anchoring dynamics implies the existence of a fast learning adaptation event (Wagman et al., 2001), as confirmed by experimental evidence of practice effects on the success of maintaining balance immediately after undergoing an anchor training protocol (Mauerberg-deCastro et al., 2012). As a dynamic system, haptic anchoring, which relies on (deliberate) exploratory activity, is a subsystem that is subservient to the postural control system (automatic and involuntary). Haptic anchoring is softly selforganized and functionally and architecturally multilayered. Such haptic perceptual organization, according to Turvey and Fonseca (2014), implies that a collective flow of tensions propagates through the varying levels of organization within the organism. Such information flow requires deformations that propagate from the individual cell to muscle cells, to the entire body, and beyond. The anchor system is an external scale of propagation of haptic deformation-a tensegrity dynamical pattern from a non-biological connector that provides information to serve a system's behavior (e.g., maintaining balance). The tensegrity components in the anchoring task form a softly assembled configuration that includes the anchor system's physical parts and the (acting) body's parts. For the sake of keeping the body's posture stable, the anchor system's softly assembled configuration allows individuals to actively move the strings to indirectly (tele) contact the surface in order to detect a spatial reference that is created by gravitational pull. Our results with the dog's walking anchoring task are good examples of how the dog's leash is an external component in the architectural haptic mechanism that combines a diverse array of tensions (i.e., the tensegrity hypothesis of Turvey and Fonseca, 2014) of two linked organisms' motions. The tension array-explained by the tensegrity hypothesis and demonstrated in the anchoring task - provides a level of task analysis that is dedicated to the haptic mechanism.

In this article, the next step in our attempt to demonstrate the haptic role of the anchor system was to justify its practical application and, therefore, determine its potential as an aid device or learning tool 
for the improvement of the postural control system. By observing groups with special needs (e.g., older individuals and intellectually disabled individuals) and the effects of intervention protocols, we preliminarily demonstrated the technological potential of the anchor system in the field of rehabilitation.

The anchor system seems to promote intrinsic adaptation at an observable level (i.e., stability changes during experimentation), as well as in non-observable ways (i.e., neural and non-neuronal plasticity). Valid intervention protocols are in demand for the treatment of neurodevelopmental disorders and other rehabilitation purposes. Further studies should expand their foci on the extent of adaptations provided by the anchor system, both short- and long-term. Through the detection of distal information mediated by objects or tools, individuals exploit information to adapt - even momentarily - their behavior to a changing environment.

We recommend that intervention studies that use the anchor system should target conditions of balance deterioration, such as in older people and individuals with neurodevelopmental disorders (e.g., cerebral palsy) and as a strategy for early intervention in a variety of disability or impairment conditions. A systematic rehabilitation program should manipulate the rate of practice, task variability, levels of task difficulty, and other factors that facilitate or compete with behavioral outcomes, such as levels of motivation, attentional factors, states of arousal, and fatigue levels, among others.

\section{Acknowledgements}

We would like to acknowledge our appreciation to the families, participants, and the special school for individuals with intellectual disability (APAE, Rio Claro) who volunteered to participate in the studies reported herein. We thank Debra Frances Campbell for her kind and patient revision of the English language. We would like to thank the $\mathrm{P} \& \mathrm{~N}$ anonymous reviewers for their insightful review and comments. We would especially like to express our gratitude to Dr. Patrick Cabe for his extensive, detailed, and thoughtful review of this paper. Finally, we would like to thank Dr. José Aparecido da Silva for the invitation to the first author to present the content of this article as a guest lecturer during the 2013 Symposium on Attention, Perception and Memory, held at the University of São Paulo, Ribeirão Preto, Brazil. Studies were supported by the National Council for Scientific and Technological Development (CNPq) and partially by the São Paulo Research Foundation (FAPESP).

\section{References}

Albertsen, I.M., Temprado, J.J., \& Berton, E. (2010). Effect of haptic supplementation on postural stabilization; A comparison of fixed and mobile support conditions. Human Movement Science, 29, 999-1010. doi:10.1016/j.humov.2010.07.013

Ando, N., Ohta, M., \& Hashimoto, H. (2000). Micro teleoperation with haptic interface. Industrial Electronics Society, 2000. IECON 2000. 26th Annual Conference of the IEEE. 1, 13-18. doi:10.1109/ IECON.2000.973119
Baccini, M., Rinaldi, L.A., Federighi, G., Vannucchi, L., Paci, M., \& Masotti, G. (2007). Effectiveness of fingertip light contact in reducing postural sway in older people. Age and Ageing, 36, 30. doi:10.1093/ageing/afl072

Baldan, A.M.S., Alouche, S.R., Araújo, I.M.G., \& Freitas, S.M.S.F. (2014). Effect of light touch on postural sway in individuals with balance problems: A systematic review. Gait and Posture, http:// dx.doi.org/10.1016/j.gaitpost.2013.12.028

Barela, J.A., Jeka, J.J., \& Clark, J.E. (1999). The use of somatosensory information during the acquisition of independent upright stance. Infant Behavior and Development, 22, 87-102. http://dx.doi. org/10.1016/S0163-6383(99)80007-1

Bolton, D.A.E., McIlroy, W.E., \& Staines, W.R. (2011). The impact of light fingertip touch on haptic cortical processing during a standing balance task. Experimental Brain Research, 212, 279. doi:10.1007/ s00221-011-2728-6.

Burton, G. (1993) Non-neuron extensions of haptic sensitivity. Ecological Psychology, 5, 105-124. doi:10.1207/ s15326969eco0502_1

Cabe, P.A. (2011). Haptic distal spatial perception mediated by strings: haptic "looming." Journal of Experimental Psychology: Human Perception and Performance, 37, 1492-1511. doi:10.1037/ a0024231

Cabe, P.A. (2013). Haptic distal spatial perception mediated by strings: Size at a distance and egocentric localization based on ellipse geometry. Attention and Perception Psychophysics, 75, 358 doi:10.3758/s13414-012-0389-6

Cabe, P.A., \& Hofman, L.L. (2012). Haptic distal spatial perception mediated by strings: Point of closest approach and bypass distance. Journal of Experimental Psychology: Human Perception and Performance, 38, 1328-1340. doi:10.1037/a0027738

Cabe, P.A., \& Pittenger, J.B. (1992). "Time-to-topple": Haptic tau. Ecological Psychology, 4, 241-246.

Calve, T., \& Mauerberg-deCastro, E. (2005). Contribuição da percepção háptica no controle postural de crianças (Haptic contribution to postural control in children). Motriz, 11, 199-204. Retrieved from http://www.rc.unesp.br/ib/efisica/motriz/11n3/ 13TCEa.pdf.

Carello, C., Silva, P.L., Kinsella-Shaw, J.M., \& Turvey, M.T. (2008). Muscle-based perception: theory, research and implications for rehabilitation. Revista Brasileira de Fisioterapia, 12, 339-350. doi:10.1590/S1413-35552008000500002

Carello, C., \& Turvey, M.T. (2004). Physics and psychology of the muscle sense. Current Directions in Psychological Science, 13, 25 28. doi:10.1111/j.0963-7214.2004.01301007.x

Chatterjee, A., Chaubey, P., Martin, J., \& Thakor, N. (2008). Testing a prosthetic haptic feedback simulator with an interactive force matching task. Journal of Prosthetics and Orthotics, 20, 27-34. doi:10.1097/01.JPO.0000311041.61628.be

Chechlacz, M., \& Gleeson, J.G. (2003). Is mental retardation a defect of synapse structure and function? Pediatric Neurology, 29, 11-17. doi:10.1016/S0887-8994(03)00152-8

Dascal, J.B., Okazaki, V.H.A., \& Mauerberg-deCastro, E. (2012). Efeitos do sistema âncora sobre o controle postural de idosos (Effects of the anchor system on postural control in older adults). Revista Brasileira de Cineantropometria e Desempenho Humano. doi:10.5007/1980-0037.2012v14n2p144

Donker, S.F., Roerdink, M., Greven, A.J., \& Beek, P.J. (2007). Regularity of center-of-pressure trajectories depends on the amount of attention invested in postural control. Experimental Brain Research, 181, 1-11. doi:10.1007/s00221-007-0905-4

Fernandez, F., \& Garner, C.C. (2007). Over-inhibition: a model for developmental intellectual disability. Trends in Neurosciences, 30 , 497-503.doi:10.1016/j.tins.2007.07.005

Freitas, M.B.Z., Mauerberg-deCastro, E., \& Moraes, R. (2013). Intermittent use of an "anchor system" improves postural control in healthy older adults. Gait and Posture, 38, 433-437. doi:10.1016/j. gaitpost.2013.01.004

Frissen, I., Ziat, M., Campion, G., Hayward, V., \& Guastavino, C. (2012). The effects of voluntary movements on auditory-haptic and haptic-haptic temporal order judgments. Acta Psychologica, 141, 140-148. doi:10.1016/j.actpsy.2012.07.010

Gibson, J.J. (1966). The senses considered as perceptual systems. Boston: Houghton Mifflin.

Gibson, J.J. (1979). The ecological approach to visual perception. Boston: Houghton Mifflin.

Holden, M., Ventura, J., \& Lackner, J.R. (1994). Stabilization of posture by precision contact of the index finger. Journal of Vestibular Research, 4, 285-301. 
Horak, F.B. (2009). Postural compensation for vestibular loss. Annals of the New York Academy of Sciences, 1164, 76-81. doi:10.1111/ j.1749-6632.2008.03708.x.

Horvat, M., Croce, R., \& Zagrodnik, J. (2010). Utilization of sensory information in intellectual disabilities. Journal of Developmental and Physical Disabilities, 22, 463-473. doi:10.1007/s10882-0099182-4

Huffman, J.L., Horslen, B.C., Carpenter, M.G., \& Adkin, A.L. (2009). Does increased postural threat lead to more conscious control of posture? Gait and Posture, 30, 528-532. doi:10.1016/j. gaitpost.2009.08.001.

Jeka, J.J. (1997). Light touch as a balance aid. Physical Therapy, 77, 476-487.

Jeka, J.J., \& Lackner, J.R. (1994). Fingertip contact influences human postural control. Experimental Brain Research, 100, 495502. Retrieved from http://link.springer.com/article/10.1007\% 2FBF00229188

Jeka, J.J., Schoner, G., Dijkstra, T., Ribeiro, P., \& Lackner, J.R. (1997). Coupling of fingertip somatosensory information to head and body sway. Experimental Brain Research, 113, 475. Retrieved from http:// djdeath.atr.free.fr/biomeca/articles/155_coupfingtip_ebr_1997.pdf.

Kim, K.K., Colgate, J. E., Santos-Munné, J.J., Makhlin, A., \& Peshkin, M.A. (2010). On the design of miniature haptic devices for upper extremity prosthetics. IEEE/ASME Transactions on Mechatronics, 15, 1-12. doi:10.1109/TMECH.2009.2013944

Kinsella-Shaw, J., \& Turvey, M.T. (1992). Haptic perception of object distance on a single-strand vibratory web. Perception and Psychophysics, 52, 625-638. Retrieved from http://link.springer. com/article/10.3758\%2FBF03211700\#

Klatzky, R.L., Lederman, S.J., \& Metzger, V.A. (1985). Identifying objects by touch: An "expert system." Perception and Psychophysics, 37, 299-302.doi:10.3758/BF03211351

Lackner, J.R., Rabin, E., \& DiZio, P. (2001). Stabilization of posture by precision touch of the index finger with rigid and flexible filaments. Experimental Brain Research, 139, 454-464. doi:10.1007/s002210100775

Lederman, S.J., \& Klatzky, R.L. (2009). Haptic perception: a tutorial. Attention, Perception, and Psychophysics, 71, 1439-1459. doi:10.3758/APP.71.7.1439

Loomis, J.M., \& Lederman, S.J. (1986). Tactual perception. In K.R. Boff, L. Kaufman, \& J.P. Thomas (Eds.), Handbook of perception and human performance (vol. 2, pp. 31-41). New York: Wiley.

Mauerberg-deCastro, E. (2004). Developing an "anchor" system to enhance postural control. Motor Control, 8, 339358. Retrieved from http://journals.humankinetics.com/ mc-backissues/MCVolume8Issue3JulyDevelopinganAnchor SystemtoEnhancePosturalControl

Mauerberg-deCastro, E., Calve, T., Viveiros, F.F., Polanczyk, S., \& Cozzani, M.V. (2003). Um tutorial sobre percepção háptica no controle postural. Ilustrando um sistema "âncora" e suas aplicações na reabilitação e na atividade física adaptada (A tutorial about haptic perception in postural control. Illustrating an "anchor" system and its applications in rehabilitation and adapted physical activity). Revista da Sociedade Brasileira de Atividade Motora Adaptada, 8, 7-20. Retrieved from http://www.rc.unesp.br/ib/ efisica/sobama/sobamaorg/vol8no12003resumos.pdf

Mauerberg-deCastro, E., Lucena, C.S., Cuba, B.W., Boni, R.C., Campbell, D.F., \& Moraes, R. (2010). Haptic stabilization of posture in adults with intellectual disabilities using a non-rigid tool. Adapted Physical Activity Quarterly, 27, 208-225. Retrieved from http://journals. humankinetics.com/apaq-back-issues/APAQVolume27Issue3July/ HapticStabilizationofPostureinAdultsWithIntellectualDisabilities UsingaNonrigidTool

Mauerberg-deCastro, E., Melo, J.A.C., \& Périco, B. (2011). Ancoragem funcional em tarefas de condução de um cão ao longo de um percurso com restrições ao sistema postural (Anchoring during restricted postural tasks while walking a dog along a pathway). Motriz, 17, S18. doi: http://dx.doi.org/10.1590/S198065742013000700009

Mauerberg-deCastro, E., Moraes, R., \& Campbell, D.F. (2012). Short-term effects of the use of non-rigid tools for postural control by adults with intellectual disabilities. Motor Control, 16, 131 Retrieved from http://journals.humankinetics.com $/ \mathrm{mc}$ back-issues/mc-volume-16-issue-2-april/short-term-effects-ofthe-use-of-non-rigid-tools-for-postural-control-by-adults-withintellectual-disabilities

Mauerberg-deCastro, E., Moraes, R., Pacheco, S.M., Costa, T.D.A., Porto, L.A., \& Pestana, M.B. (2013a). Haptic posture stabilization via "light touch" and via the "anchor system": effects of contact points. Motriz, 19, S177. Retrieved from http://www.periodicos. rc.biblioteca.unesp.br/index.php/motriz/article/view/7469/pdf

Mauerberg-deCastro, E., Moraes, R., Tavares, C.P., Pestana, M.B., Porto, L.A., \& Dias, M.S. (2013b). Usefulness of haptic information to individuals in a quiet balance position, provided by holding a leash attached to a dog walking on a treadmill Retrieved from http:/www.periodicos.rc.biblioteca.unesp.br/index.php/motriz/ article/view/7469/pdf

Mauerberg-deCastro, E., Tavares, C.P., Costa, T.D.A., Périco, B.C., Pestana, M.B., \& Porto, L.A. (2013c). Human postural stability during dog walking by adults with intellectual disability. Hacettepe Journal of Sport Sciences, 24, 139-142. Retrieved from http://www.sbd.hacettepe.edu.tr/index.php? pid $=2025$ \&aid $=332$

Maurer, C., \& Peterka, R.J. (2005). A new interpretation of spontaneous sway measures based on a simple model of human postural control. Journal of Neurophysiology, 93, 189-200. doi:10.1152/jn.00221.2004

Michaels, C.F., \& Carello, C. (1981). Direct perception. Englewood Cliffs: Prentice-Hall. Retrieved from http://ione.psy.uconn. edu/ cespaweb/docs/MC.pdf

Molofsky, A.V., Krenick, R., Ullian, E., Tsai, H., Deneen, B., Richardson, W.D., ... Rowitch, D.H. (2012). Astrocytes and disease: a neurodevelopmental perspective. Genes and Development, 26 , 891-907. doi:10.1101/gad.188326.112

Moraes, R., \& Mauerberg-deCastro, E. (2009). O uso de ferramenta não-rígida reduz a oscilação corporal em indivíduos idosos (The use of non-rigid tools reduce postural sway in older individuals). Motriz, 15, 263-272. Retrieved from http://www.periodicos. rc.biblioteca.unesp.br/index.php/motriz/article/view/2373/2295

National Research Council (1997). Advanced technology for human support in space. Washington, DC: National Academy Press. Retrieved from http://www.nap.edu/openbook.php?record $\mathrm{id}=5826 \&$ page $=\mathrm{R} 1>$

Okamura, A.M. (2009). Haptic feedback in robot-assisted minimally invasive surgery. Current Opinion in Urology, 19, 102 doi:10.1097/ MOU.0b013e32831a478c

Périco, B.C., Mauerberg-deCastro, E., Pestana, M.B., Porto, L.A., Magre, F.L., \& Pacheco, S.C.M. (2013). Estabilidade locomotora durante a condução de um cão (Locomotor stability while walking a dog). Motriz, 19, S57-S67. doi:10.1590/S1980-65742013000700009.

Petrov,A.A., Dosher, B.A., \& Lu,Z.L.(2005). The dynamics of perceptual learning: an incremental reweighting model. Psychological Review, 112, 715-743. doi: 10.1037/0033-295X.112.4.715.

Pettersson, A.F., Olsson, E., \& Wahlund, L.O. (2007). Effect of divided attention on gait in subjects with and without cognitive impairment. Journal of Geriatric Psychiatry and Neurology, 20, 58-62. doi:10.1177/0891988706293528

Polanczyk, S.D. (2003). Utilidade do paradigma "âncora” no controle postural e nas estratégias de mobilidade durante sessões de atividade fisica adaptada para individuos com deficiência mental (Usefulness of the "anchor" paradigm in postural control and mobility strategies during physical activity sessions in individuals with intellectual disabilities) (Unpublished monograph). Rio Claro: São Paulo State University.

Rabin, E., Chen, J., Muratori, L., DiFrancisco-Donoghue, J., \& Werner, W.G. (2013). Haptic feedback from manual contact improves balance control in people with Parkinson's disease. Gait and Posture, 38, 373-379. doi:10.1016/j.gaitpost.2012. 12.008.

Rabin, E., DiZio, P., \& Lackner, J.R. (2006). Time course of haptic stabilization of posture. Experimental Brain Research, 170, 122 doi:10.1007/s00221-006-0348-3.

Riccio, G.E., \& Stoffregen, T.A. (1988). Affordances as constraints on the control of stance. Human Movement Science, 7, 265-300. doi:10.1016/0167-9457(88)90014-0

Riley, M.A., Stoffregen, T.A., Grocki, M.J., \& Turvey, M.T. (1999). Postural stabilization for the control of touching. Human Movement Science, 18, 795-817. doi:10.1016/S0167-9457(99)00041-X

Runeson, S. (1977). On the possibility of "smart" perceptual mechanisms. Scandinavian Journal of Psychology, 18, 172-179. doi:10.1111/j.1467-9450.1977.tb00274.x

Shumway-Cook, A., \& Woollacott, M. (1985). Dynamics of postural control in the child with Down syndrome. Physical Therapy, 65, 1315-1322. Retrieved from http://ptjournal.apta.org/ content $/ 65 / 9 / 1315$

Solomon, H.Y., \& Turvey, M.T. (1988). Haptically perceiving the distances reachable with hand-held objects. Journal of 
Experimental Psychology and Human Perception Performance, 14, 404-427. doi:10.1037/0096-1523.14.3.404

Stoffregen, T.A., Smart, L.J., Bardy, B.G., \& Pagulayan, R.J. (1999). Postural stabilization of looking. Journal of Experimental Psychology: Human Perception and Performance, 25, 1641-1658. doi:10.1037/0096-1523.25.6.1641

Tibbetts, P.E. (2004). The concept of voluntary motor control in the recent neuroscientific literature. Synthese, 141, 247-276. Retrieved from http://philpapers.org/rec/TIBTCO>

Turvey, M.T. (1996). Dynamic touch. American Psychologist, 51, 1134-1152. doi:10.1037/0003-066X.51.11.1134

Turvey, M.T., \& Fonseca, S.T. (2014). The medium of haptic perception: A tensegrity hypothesis. Journal of Motor Behavior, 46, 143-187. http://dx.doi.org/10.1080/00222895.2013.798252

Uyanik, M., \& Kayihan, H. (2010). Down syndrome: Sensory integration, vestibular stimulation and neurodevelopmental therapy approaches for children. In: J.H. Stone, \& M. Blouin (Eds.), International Encyclopedia of Rehabilitation. Center for International Rehabilitation Research Information and Exchange. Retrieved from http://cirrie.buffalo.edu/encyclopedia/en/article/48

Vaillend, C., Poirier, R., \& Laroche, S. (2008). Genes, plasticity and mental retardation. Behavioural Brain Research, 192, 88-105. doi:10.1016/j.bbr.2008.01.009

Von Fieandt, K. (1966). The world of perception. Homewood, IL: Dorsey Press.

Vuillerme, N., Isableu, B., \& Nougier, V. (2006). Attentional demands associated with the use of a light fingertip touch for postural control during quiet standing. Experimental Brain Research, 169, 232-236. doi:10.1007/s00221-005-0142-7

Wagman, J.B., Shockley, K., Riley, M.A., \& Turvey, M. T. (2001). Attunement, calibration, and exploration in fast haptic perceptual learning. Journal of Motor Behavior, 33, 323-327. 